\title{
Oxitocina intrafunicular Vs. sistémica en el manejo de la tercera etapa del parto eutócico
}

\author{
Mike J. Castañeda C.*; Armando Hernández Rojas**
}

\begin{abstract}
RESUMEN: Durante la tercera etapa del parto, el alumbramiento se presenta la mayoría de la morbilidad por hemorragia, muchos autores han utilizado por vía intrafunicular oxitocina para su manejo y para el de la retención placentaria, con resultados positivos y negativos. Nosotros tratamos de demostrar si en realidad la oxitocina por vía intrafunicular modificaba en grado alguno el alumbramiento, durante partos eutócicos, encontrando que es el volumen inyectado del vehículo (SSN) y no el efecto farmacológico de la oxitocina lo que contribuye a la disminución del tiempo y de sangrado.
\end{abstract}

PALABRAS CLAVES: Oxitocina intraumbilical, alumbramiento, Parto eutócico.

SUMMARY: The third stage of labor presents the majority bleeding morbidity, and many autors have used intrafunicular oxitocin for its management and the retained placenta, with positive and negative results. The pourpose of the study was to determine if intrafunicular oxitocin modifes the third stage of labor, during a normal labor. We found that the volume, and not the farmacology action of oxitocin was the reason for the diminish bleeding and third stage of labor time.

KEY WORDS: Intrafunicular oxitocin, third stage of labor, normal labor.

Golan y cols. describieron un método para el manejo de placentas retenidas, inyectando 10 unidades de oxitocina diluidas en $20 \mathrm{cc}$ de solución salina normal por vía intrafunicular en placentas que estaban retenidas por más de treinta minutos, todas alumbraron espontáneamente a los tres minutos y cuarenta segundos en promedio. Posteriormente Kristiansen y cols, Chestnut y Wilcox, Porter y cols y otros autores han presentado resultados positivos y negativos en el uso intrafunicular de la oxitocina. Reddy y Carey utilizaron la oxitocina intrafunicular como alternativa para el manejo del alumbramiento en partos normales, encontrando acortamiento en el tiempo de alumbramiento y en el volumen de sangrado en las pacientes manejadas con oxitocina intrafunicular. En este último trabajo se compararon dos vías de aplicación, intrafunicular y endovenosa, hallando ventajas en la primera en un grupo de 50 pacientes. Nosotros tratamos de comparar la vía endovenosa, con la vía intrafunicular agregando un tercer grupo de pacientes que se manejaron intrafunicularmente solo con solución salina normal. El objetivo del presente trabajo era comprobar la disminución en el tiempo de alumbramiento y volumen de hemorragia con el uso de oxitocina intrafunicular.

\section{Materiales y métodos}

Se estudiaron 42 pacientes que cumplieron los criterios de inclusión. Se excluyeron las pacientes que no

Especialista Sección Ginecología y Obstetricia Hospital Militar Central.

Residente III Ginecología y Obstetricia Universidad Militar Nueva Granada- Hospital Militar Central. presentaban partos normales, pretérmino o postérmino, partos inducidos, pacientes con patología asociada a la gestación o fuera de ella, pacientes grandes multíparas, las que recibían sulfato de magnesio u oxitocina previas al parto, las madres RH negativas, pacientes con antecedente de cesárea o cirugía uterina, y las que no estaban conformes con el procedimiento. A todas las pacientes con criterios de inclusión se les tomó muestra para hemoglobina y hematocrito previos al parto y 8 a 12 horas postparto. Luego de el nacimiento del niño se contabilizó el tiempo del alumbramiento, y se manejó el alumbramiento en tres modalidades: A-10 unidades de oxitocina diluidas en $20 \mathrm{cc}$ de solución salina normal por vía intrafunicular. B- $20 \mathrm{cc}$ de solución salina normal por vía intrafunicular. C- oxitocina por vía endovenosa $5 \mathrm{u}$ directas y 5 en goteo.

Los datos fueron recogidos en el lapso octubre 1995 a octubre de 1996, los partos fueron supervisados o atendidos por los investigadores. La inyección intrafunicular en los grupos A y B se realiza inmediatamente al pinzamiento del cordón, y posteriormente se "exprime" hasta el introito donde se vuelve a pinzar y se espera el alumbramiento.

\section{Resultados}

Los resultados se resumen en la tabla 1. En los tres grupos de pacientes fueron similares la paridad, el peso placentario y el tiempo de los dos primeros periodos del parto.

El tiempo de el tercer período del parto fue menor en los grupos manejados intrafunicularmente (grupos A y B) comparado con el manejo intravenoso (grupo C), al igual que la diferencia entre los hematocritos preparto y 
Tabla 1

OXITOCINA INTRAFUNICULAR VS SISTEMICA EN EL MANEJO DE LA TERCERA ETAPA DEL PARTO EUTOCICO, RESULTADOS

\begin{tabular}{|c|c|c|c|c|c|c|}
\hline Grupo & $\begin{array}{c}\text { Edad } \\
\text { Primer } \\
\text { Período Hrs }\end{array}$ & $\begin{array}{c}\text { Tiempo } \\
\text { Expulsivo } \\
\text { Minutos }\end{array}$ & $\begin{array}{c}\text { Tiempo } \\
\text { Grs }\end{array}$ & $\begin{array}{c}\text { Peso placenta } \\
\text { Alumbramiento } \\
\text { Minutos }\end{array}$ & Tiempo & Caída Hto. \\
\hline A & 25.2 & 5.8 & 31.9 & 527.7 & 2.6 & 4.0 \\
B & 25.6 & 6.8 & 28.9 & 519.3 & 2.5 & 3.9 \\
C & 25.8 & 6.5 & 33.3 & 492.0 & 6.0 & 6.3 \\
\hline
\end{tabular}

postparto. La diferencia en tiempo fue estadísticamente significativa comparando los grupos A-C (p:0,002) y BC (p:0,001), pero no entre los grupos A-B (p:0,7). La diferencia en la caída del hematocrito solo fue significativa cuando se compararon los grupos B-C (p:0,018) (Tabla 2).

En ninguna paciente hubo retención placentaria, ni se requirió la extracción manual de la placenta. Igualmente no hubo complicaciones hemorrágicas en ninguno de los grupos ni se requirió la transfusión de sangre postparto, todas las pacientes evolucionaron satisfactoriamente.

\section{Discusión}

En la literatura se encuentran resultados que muestran las bondades del uso de la oxitocina intrafunicular en el manejo de la tercera etapa del parto, y en el manejo de la placenta retenida, como también estudios que no hallan valor a esta aplicación. Nosotros encontramos diferencias entre el manejo intrafunicular vs sistémico del alumbramiento, pero no hay significancia estadística entre el uso de oxitocina intrafunicular o el de solución salina sola, lo que nos sugiere que es el efecto mecánico y no la acción farmacológica de la oxitocina el que favorece la disminución en tiempo y sangrado durante el alumbramiento.

Aunque se demuestra significancia estadística, clínicamente la intervención intrafunicular del alumbramiento no se favorece de manera importante.

Tabla 2

OXITOCINA INTRAFUNICULAR VS SISTEMICA EN

EL MANEJO DE LA TERCERA

ETAPA DEL PARTO EUTOCICO. CORRELACION ( T student)

\begin{tabular}{|l|c|c|}
\hline Grupo & $\begin{array}{c}\text { Tiempo } \\
\text { alumbramiento }\end{array}$ & $\begin{array}{c}\text { Caída } \\
\text { hematocrito }\end{array}$ \\
\hline A vs B & P: $0.76(\mathrm{NS})$ & P: 0.17 (NS) \\
A vs C & P: 0.002 & P: $0.23(\mathrm{NS})$ \\
B vs C & P: 0.001 & P: 0.018 \\
\hline
\end{tabular}

\section{BIBLIOGRAFIA}

1. Cifuentes R. Obstetricia de alto riesgo 1994; 139-142.

2. Chestnut DH et al: Influence of umbilical vein administration of oxitocin on third stage of labor: a randomized, double blind, placebocontrolled study. Am. J. Obstet. Gynaecol. 1987; 157:160-162.

3. Decherney: Current in Obstet \& Gynecol. 8a. de 1994.

4. Golan A: Intraumbilical oxitocin for the retained placenta. Am. J. Obstet. Gynaecol. 1988; 159: 1309-1310.

5. Huber MC et al: Umbilical vein administration of oxitocin for the management of the retained placenta: is it effective?. Am. J. Obstet. Gynaecol. 1991; 164: 1216-1219.

6. Kristiansen FV et al: The effect of oxitocin injection into the umbilical vein for the management of the retained placenta. Am. J. Obstet. Gynaecol. 1987; 156: 979-980.
7. Pipingas A et al: Umbilical vassel oxitocin administration for retained placenta: in vitro study of varius infussion techniques. Am. J. Obstet. Gynaecol. 1993; 160: 793-795.

8. Porter KD et al. A randomized comparison of umbilical vein and intravenus oxitocin during the puerperium. Obstet. Gynaecol. 1991; 70: 254-256.

9. Reddy V Carey C: Effect of umbilical vein oxitocin on puerperal blood loss and length of the third stage of labor. Am. J. Obstet. Gynaecol. 1989; 160: 206-208. 